\title{
Do food additives cause hyperactivity in preschool children?
}

Bateman B, Warner JO, Hutchinson E, Dean T, Rowlandson P, Gant C, et al. The effects of a double blind, placebo controlled, artificial food colourings and benzoate preservative challenge on hyperactivity in a general population sample of preschool children. Arch Dis Child 2004;89:506-11.

Background: There have been claims that artificial food additives such as colouring and benzoate preservatives are potentially linked to hyperactivity in children ${ }^{1,2}$ and that children with atopy may be at particular risk. ${ }^{3}$ However, the generalizability of findings from these studies is limited because of the small, highly selected samples.

Design: Parents of all 3-year-old children living on the Isle of Wight, UK, were sent a letter inviting them to participate in the trial. Of the 2878 children whose parents were contacted, 277 completed the randomized, placebo-controlled, doubleblind, crossover study. Children were entered into 1 of 4 groups based on the presence or absence of hyperactivity and atopy. The study took place over 4 weeks, during which the participants were instructed to fol-

$\infty$ low an additive-free diet. The first and third weeks were unblinded washout periods. In the second and fourth weeks, the children received bottles of fruit juice (placebo challenge) or fruit juice containing known quantities of food additives (active

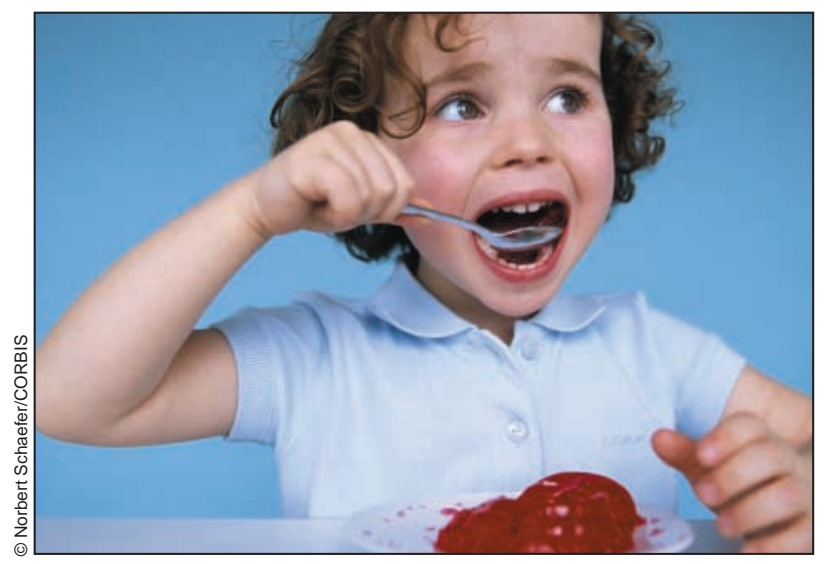

challenge). Research psychologists assessed the children's behaviour weekly using validated tests. In addition, the parents rated behaviour daily and kept a diary detailing consumption of the provided drinks and compliance with the diet.

Results: The assessments by the research psychologists showed no change in hyperactive behaviour resulting from the addition of or washout from the food additives. By contrast, parental ratings showed decreases in hyperactive behaviour when the food additives were limited (i.e., during the washout periods from the baseline normal diet and between the placebo and active challenges) and increases in hyperactive behaviour during the placebo and active challenge periods. However, these increases in hyperactivity did not deviate significantly from baseline measures. The child's initial hyperactivity level did not have an effect on the parental ratings. There was no association observed between atopy or preexisting hyperactivity and hyperactivity during the study period.

Commentary: Although the authors attempted to conduct a population-based randomized controlled trial, they were able to recruit only 397 of the 2878 children 3 years of age on the Isle of Wight. No comparison was made between the 277 participants who completed the trial and the 2581 eligible children who did not. Indeed, given the demands placed on both subjects and their parents to complete the study's protocol, parents whose children completed the study may have represented a bi- ased sample who had preexisting notions on the behavioural effects of food additives. Therefore, it is unlikely that the findings are generalizable to the population studied, let alone to other populations.

Still, the parents found that the removal of artificial food additives from a child's diet may have a beneficial effect on hyperactive behaviour. However, parents were not blinded to the removal of food additives, and the research psychologists detected no benefit from such removal. The challenge with the food additives did not appear to differ from the placebo challenge in its effects on hyperactive behaviour according to the parental ratings.

Practice implications: This trial is the most rigourous test of the effect of artificial food additives on hyperactive behaviour in preschool children to date. However, it remains unclear whether the removal or controlled addition of food additives has an effect on hyperactive behaviour. There are still insufficient grounds to advise parents to attempt to limit the exposure to food additives in the diets of their children.

Paul A. Kurdyak

Research Fellow

Centre for Addiction and Mental

Health

Department of Psychiatry

University of Toronto

Toronto, Ont.

\section{References}

1. Conners CK, Goyette CH, Southwick DA. Food additives and hyperkinesis: preliminary report of a double-blind crossover experiment. Psychopharmacol Bull 1976;12(2):10-1.

2. Weiss B, Williams JH, Margen S, Abrams B, Caan B, Citron LJ, et al. Behavioral responses to artificial food colors. Science 1980;207:1487-9.

3. Carter CM, Urbanowicz M, Hemsley R, Mantilla L, Strobel S, Graham PJ, et al. Effects of a few food diet in attention deficit disorder. Arch Dis Child 1993;69(5):564-8. 